\title{
$A b$ Initio HF and DFT Calculation of the Second Order NLO Response of Tetrathiafulvalene and 1,3-Dithiole Derivatives
}

\author{
R. Andreu, M. J. Blesa, S. Franco, J. Garín, J. Orduna* \\ Departamento de Química Orgánica-ICMA. Universidad de Zaragoza-CSIC \\ E-50009 Zaragoza, Spain
}

\begin{abstract}
The nonlinear optical properties of new tetrathiafulvalenes and 1,3-dithioles have been studied using first principle methods. The higher hyperpolarizabilities of the extended 1,3-dithioles compared to Tetrathiafulvalene analogs are explained on the basis of the less localized HOMO of the former giving rise to higher transition dipole moments and a more pronounced decrease of the HOMO-LUMO gap on chain extension.
\end{abstract}

Keywords: Ab initio, Density Functional Theory, Nonlinear optical materials. PACS: 31.15Ar, 31.15EW, 42.70Ng.

\section{INTRODUCTION}

As a continuation of our work in the search for new organic molecules exhibiting high molecular hyperpolarizabilities, we have recently reported ${ }^{1}$ the synthesis and nonlinear optical properties of new dipolar NLO active molecules having the closely related 1,3-dithiole or tetrathiafulvalene donors and 1,1,3-tricyano-2phenylpropene as an acceptor. The experimental data revealed that lengthening of the ethylenic spacer in the TTF series 2 increases the $\mu \beta(0)$ product from $225 \cdot 10^{-48}$ esu to $520 \cdot 10^{-48}$ esu on passing from $\mathbf{2 a}$ to $\mathbf{2 b}$ but further increase of the conjugated path in $\mathbf{2 c}$ does not lead to an increased NLO response. The 1,3-dithiole series $\mathbf{1}$, on the other hand, displays a continuous enhancement of the hyperpolarizability on lengthening the $\pi$ spacer and compound $1 \mathbf{d}$ reaches a $\mu \beta(0)$ value of $3675 \cdot 10^{-48}$ esu, exceeding the maximum NLO response reported up to date for any $1,3-$ dithiole or thetrathiafulvalene derivative. The unusual behaviour of tetrathiafulvalenes is at least partially caused by the lack of the bathochromic shift expected for the higher homologues in this series that has been already observed in TTFs conjugated to strong acceptor groups. ${ }^{2}$<smiles>[R]C1=C([R])SC(=C(C)CC)S1</smiles>

1 $a, n=0 ; b, n=1 ; c, n=2 ; d, n=3$.<smiles>C=CC1=CSC(=C2SC=CS2)S1</smiles>

2

$a, n=0 ; b, n=1 ; c, n=2$.

Figure 1. Studied molecules. 
We have performed $a b$ initio Hartree-Fock (HF) and density functional theory (DFT) calculations with the aim to rationalize the much better $\pi$-donor ability of 1,3-dithiole compared to the better electronic donor TTF and to get further insight into the unusual linear and nonlinear optical behaviour of tetrathiafulvalenes.

\section{RESULTS AND DISCUSSION}

The molecular geometry of the studied compounds has been optimized using the B3P86/6-31G* model chemistry, without any restriction. The proximity of the phenyl ring to one of the cyano groups forces these molecules to adopt a nonplanar geometry with the phenyl group rotated $c a 50^{\circ}$ from the molecular plane and the dicyanovinyl group rotated by $c a 10^{\circ}$. Furthermore, tetrathiafulvalene in compounds 2 arranges in a boat conformation $^{3}$ due to folding at the sulphur atoms (see figure 2).

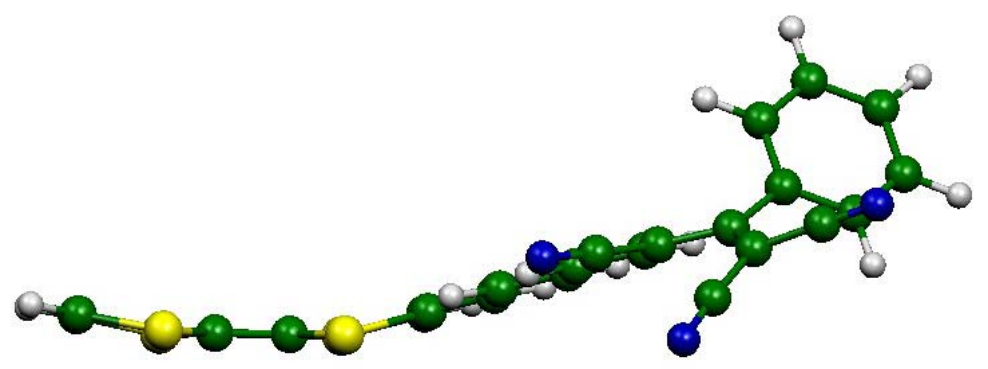

Figure 2. Optimized geometry of 2c.

The Bond Length Alternation (BLA) defined as the average difference between double and single bond lengths provides a useful estimation of the polarization of a molecule. According to Marder et al. ${ }^{4}$, the optimum NLO response is obtained at a BLA of $\pm 0.05 \AA$. The calculated BLA for $\mathbf{1 b}, \mathbf{1 c}$ and $\mathbf{1 d}$ is $-0.031,-0.039$ and $-0.041 \AA$ respectively, while the BLA for $\mathbf{2 a}, \mathbf{2} \mathbf{b}$ and $\mathbf{2 c}$ is in every case $-0.053 \AA$, indicating that tetrathiafulvalenes $\mathbf{2}$ are in every case near the optimum polarization. By contrast, 1,3-dithioles $\mathbf{1}$ are too polarized to obtain the maximum hyperpolarizability but lengthening of the conjugated spacer causes a decreased ground state charge transfer and therefore approaches to the optimum BLA.

Considering the poor reliability of DFT methods ${ }^{5}$ in the calculation of nonlinear optical properties, molecular hyperpolarizabilities were calculated using the Coupled Perturbed Hartree-Fock (CPHF) method and are gathered in table 1. It can be seen that there is a reasonable agreement between theoretical calculations and experimental results for most of the studied compounds, however, CPHF is not able to predict the decrease in $\mu \beta(0)$ on passing from $\mathbf{2 b}$ to $2 c$.

TABLE 1. Experimental values and results of theoretical calculations.

\begin{tabular}{lcccccc}
\hline Compound & \multicolumn{2}{c}{ Experimental Values $^{\mathbf{a}}$} & \multicolumn{4}{c}{ Theoretical Calculations } \\
& $\mathrm{E}_{01}(\mathrm{eV})$ & $\mu \beta(0)\left(10^{-48} \mathrm{esu}\right)^{\mathrm{b}}$ & $\mathrm{E}_{01}(\mathrm{eV})^{\mathrm{c}}$ & $\Delta \mu_{01}(\mathrm{D})^{\mathrm{c}}$ & $\mu_{01}(\mathrm{D})^{\mathrm{c}}$ & $\mu \beta(0)\left(10^{-48} \mathrm{esu}\right)^{\mathrm{d}}$ \\
\hline $\mathbf{1 a}^{\mathbf{e}}$ & 2.66 & 40 & 2.95 & 8.34 & 4.96 & -9 \\
$\mathbf{1 b}^{\mathbf{f}}$ & 2.10 & 105 & 2.84 & 6.86 & 9.19 & 194 \\
$\mathbf{1 c}^{\mathbf{f}}$ & 1.80 & 935 & 2.59 & 10.17 & 11.68 & 979 \\
$\mathbf{1 d}^{\mathrm{f}}$ & 1.74 & 3675 & 2.37 & 13.70 & 13.91 & 2531 \\
$\mathbf{2 a}$ & 1.72 & 225 & 1.49 & 21.24 & 5.11 & 292 \\
$\mathbf{2 b}$ & 1.75 & 520 & 1.47 & 25.71 & 5.97 & 654 \\
$\mathbf{2 c}$ & 1.80 & 463 & 1.44 & 31.27 & 6.93 & 1354 \\
\hline
\end{tabular}

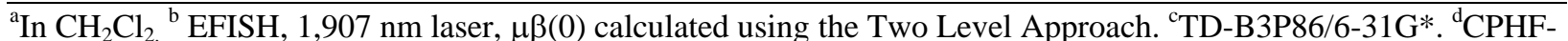
6-31G*. ${ }^{\mathrm{e}} \mathrm{R}=\mathrm{H}$. ${ }^{\mathrm{f}}$ Experimental values for $\mathrm{R}=\mathrm{CH} 3$, calculations with $\mathrm{R}=\mathrm{H}$.

A more intuitive understanding of the NLO response can be obtained from the Two Level Approach that assumes that most of the total hyperpolarizability arises from the electronic transition from the ground state to the first excited state and is given by the following expression ${ }^{6}$ : 


$$
\beta(0) \propto \frac{\Delta \mu_{01} \mu_{01}^{2}}{E_{01}^{2}}
$$

Were $\beta(0)$ is the zero-frequency hyperpolarizability, $\Delta \mu_{01}$ the dipole moment change on excitation, $\mu_{01}$ the transition dipole moment and $E_{01}$ the excitation energy. The parameters involved in this equation have been calculated using TD-DFT calculations and are also reported in table 1.

According to these calculations the lowest allowed electronic excitation causes, in every case, a one electron transition from the HOMO to the LUMO. The agreement between the calculated and observed excitation energies is only moderate, probably due to the poor ability of TD-DFT to predict the energies of transitions involving intramolecular charge transfer ${ }^{7}$ and to solvent effects that have not been considered in the calculations. However, TD-DFT reproduces the experimental trends showing a clear bathochromic shift on lengthening the $\pi$ spacer in compounds 1, while the excitation energy is almost unaltered along the TTF series 2. Quite surprisingly, these tetrathiafulvale derivatives reproduce the optical behaviour of metalorganic compounds with NLO activity. ${ }^{8}$ The HOMO mainly contributed from $d$ orbitals localized on the metal center and not interacting with the spacer explained the unaltered HOMO-LUMO gap and therefore the lack of bathochromic shift on lengthening the spacer in the metalorganic compounds, and the HOMO highly localized on the tetrathiafulvalene moiety (see figure 3) could explain the same effect in TTFs 2 .

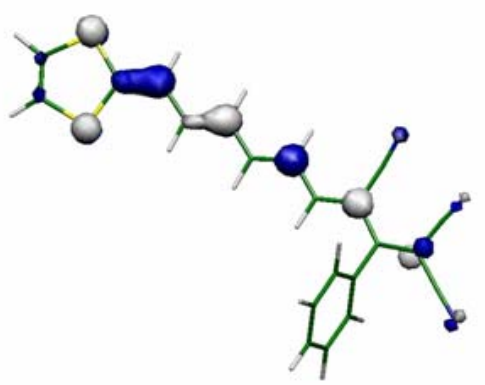

HOMO: $-6.12 \mathrm{eV}$

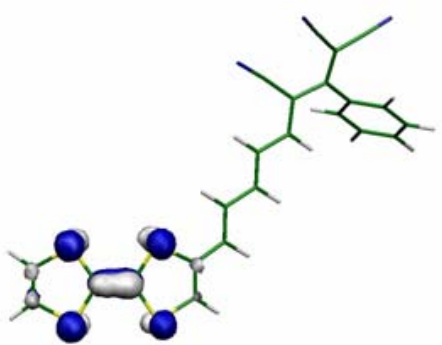

HOMO: $-5.75 \mathrm{eV}$

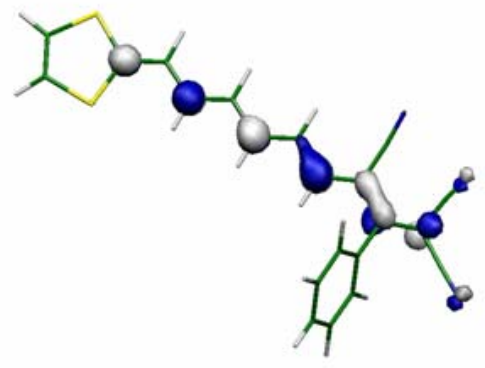

LUMO: $-3.86 \mathrm{eV}$

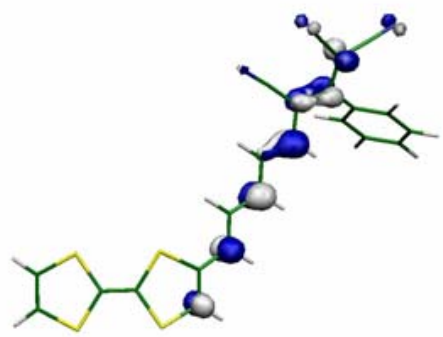

LUMO: $-4.05 \mathrm{eV}$

Figure 3. Contour plots of the HOMO and LUMO of 1d (top) and 2c (bottom).

A most important difference between 1,3-dithioles and tetrathiafulvalenes is shown in the calculated transition dipole moments. While the transition dipole steadily increases along the 1,3-dithiole series, it only changes $1.82 \mathrm{D}$ on passing from $\mathbf{2 a}$ to $\mathbf{2 c}$, and the maximum value along the TTF series $(6.93 \mathrm{D})$ is comparable to that of the lower homologues in series $\mathbf{1}$.

The highly localized HOMO in tetrathiafulvalene scarcely overlaps the LUMO that extends from the acceptor along the ethylenic spacer to the first carbon atoms in the TTF ring (Figure 3). Since HOMO-LUMO overlap is a prerequisite to obtain high transition dipoles, TTF derivatives do not give rise to high transition dipoles and therefore large hyperpolarizabilities cannot be expected for these compounds.

A completely different picture is found in 1,3-dithiole derivatives $\mathbf{1}$ for which both the HOMO and the LUMO extend along the ethylenic bridge giving rise to a large HOMO-LUMO overlap and therefore high transition dipoles. 
The higher localization of the HOMO in the tetrathiafulvalenes compared to 1,3-dithioles is responsible for larger charge displacements and hence larger dipole moment changes on excitation, and although this fact favours the NLO properties of TTFs with respect to 1,3-dithioles it cannot overcome the larger effect of the transition dipole moments on the molecular hyperpolarizabilities.

\section{CONCLUSSION}

The calculation of the parameters involved in the Two Level Approach gives rise to the expected trends in 1,3dithioles and the extension of the ethylenic spacer gives rise to a bathochromic shift, and increased transition dipole moments and dipole moment changes. Tetrathiafulvalenes, on the other hand, show quite surprising trends since extension of conjugation gives rise to only minor changes in the excitation energies and transition dipoles. This unusual behaviour can be explained on the basis of highly localized HOMOs that give rise to a poor interaction with the ethylenic bridge and the acceptor.

It can be concluded that tetratiafulvalene is not an adequate donor in the search for chromophores with very high molecular hyperpolarizabilities.

\section{ACKNOWLEDGMENTS}

Financial support from MEC-FEDER (CTQ2005-01368) and Gobierno de Aragón-Fondo Social europeo (E-39) is acknowledged.

\section{REFERENCES}

1. S. Alías, R. Andreu, M. J. Blesa, S. Franco, J. Garín, A. Gragera, J. Orduna, P. Romero, B. Villacampa and M. Allain, J. Org. Chem., In Press (2007).

2. (a) M. González, N. Martin, J. L. Segura, C. Seoane, J. Garín, J. Orduna, R. Alcalá, C. Sánchez and B. Villacampa. Tetrahedron Lett., 40, 8599-8602 (1999). (b) J. Garín, J. Orduna, J. I. Rupérez, R. Alcalá, B. Villacampa, C. Sánchez, N. Martín, J. L. Segura, M. González, Tetrahedron Lett., 39, 3577-3580 (1998).

3. (a) R. Viruela, P. M. Viruela, R. Pou-Amérigo, E. Ortí, Synth. Met., 103, 1991-1992 (1999). (b) E. Demiralp, W. A. Goddard III. J. Phys. Chem. A, 101, 8128-8131 (1997).

4. (a) S. R. Marder, L.-T. Cheng, B. G. Tiemann, A. C. Friedli, M. Blanchard-Desce, J. W., Perry, J. Skindhøj, Science 263, 511-514 (1994). (b) M. Blanchard-Desce, V. Alain, P. V. Bedworth,. S. R. Marder, A. Fort, C. Runser, M. Barzoukas, S. Lebus, R. Wortmann, Chem. Eur. J. 3, 1091-1104 (1997).

5. B. Champagne, E. A. Perpète, D. Jacquemin, S. J. A.Van Gisbergen, E.-J. Baerends, C.Soubra-Ghaoui, K. A. Robins, B. Kirtman, J. Phys. Chem. 104, 4755-4763 (2000).

6. (a) J. L. Oudar, D. S. Chemla, J. Chem. Phys., 66, 2664-2668 (1977). (b) J. L. Oudar, J. Chem. Phys., 67, $446-457$ (1977).

7. D. J. Tozer, R. D. Amos, N. C. Handy, B. O. Roos, L. Serrano-Andrés, Mol. Phys., 97, 859-868 (1999).

8. (a) B. J. Coe, L. A. Jones, J. A. harris, B. S. Brunschwig, I. Asselberghs, K. Clays, A. Persoons, J. Garín and J. Orduna, J. Am. Chem. Soc., 126, 3880-3891 (2004). (b) S. barlow, H. E. Bunting, C. Ringham, J. C. Green, G. U. Bublitz, S. G. Boxer, J. W. Perry, S. R. Marder, J. Am. Chem. Soc., 121, 3715-3723 (1999). 